\title{
New approaches in the treatment of short term and middle of the night insomnia: emerging evidence for a role for sublingual zolpidem tablets
}

\author{
This article was published in the following Dove Press journal: \\ Nature and Science of Sleep \\ 13 April 2010 \\ Number of times this article has been viewed
}

\author{
Raymond L Ownby \\ Department of Psychiatry \\ and Behavioral Medicine, \\ Nova Southeastern University, \\ Fort Lauderdale, Florida, USA
}

Correspondence: Raymond L Ownby 3200 South University Drive, Fort Lauderdale FL, 33328, USA

Tel + |-954-262-|48|

Email ro7ı@ nova.edu

\begin{abstract}
Insomnia affects a significant proportion of the general population and an even greater proportion of patients seen in general medical care. Insomnia has multiple negative effects on health status, decreases quality of life, and is associated with increased health care costs. Current treatments for insomnia include pharmacologic and behavioral strategies. Pharmacologic treatments may be effective for short-term and middle-of-the-night (MOTN) insomnia, but the usefulness of many sleep medications is limited by the residual daytime sedation with which they are associated. This problem is especially important in the case of MOTN insomnia, when only a few hours may elapse between the time a patient takes the medication and when he or she must rise. The development of sublingual and low-dose formulations of zolpidem raises the possibility that pharmacologic therapy may allow patients with MOTN insomnia to be effectively treated with a decreased risk of residual daytime sedation. Current studies of this strategy are promising, and several formulations are in the process of being brought to market.
\end{abstract}

Keywords: zolpidem, eszopiclone, zaleplon, insomnia

Insomnia, defined as difficulty in initiating and maintaining sleep or as having nonrestorative sleep, is a common problem with significant consequences for workplace productivity, public safety, and health. ${ }^{1}$ Although studies of insomnia prevalence have varied at least in part due to methodological differences, the report of the 2005 State of the Science conference convened by the US National Institutes of Health ${ }^{2}$ concluded that about $30 \%$ of the population reports sleep complaints and that $10 \%$ have symptoms of daytime impairment from insomnia. In clinical populations the prevalence of insomnia is much greater and is as high as $50 \% .^{2}$ More recently, a population-based poll by the US National Sleep Foundation found that significant numbers of persons report sleep difficulties. Almost one-third (29\%) reported having difficulties in getting to sleep at least a few nights each week. ${ }^{3}$ Twenty-eight percent of respondents in this poll reported that they were awake "a lot" during the night almost every night, while another $18 \%$ reported a similar difficulty at least a few nights a week. Insomnia has profound functional and economic consequences for those affected and for society. It is associated with significantly increased health care costs ${ }^{4}$ and has important effects on patients' daytime functioning. ${ }^{5}$ In the elderly, insomnia is associated with the risk of development and for persistent depression ${ }^{6,7}$ and increased risk of mortality. ${ }^{8}$ In spite of varying estimates and the difficulties in ascertainment associated with self report of symptoms, it is clear that insomnia is a common problem that likely affects millions of persons. 
Insomnia is generally defined by the patients' complaints of persistent difficulties in initiating or maintaining sleep or in having nonrestorative sleep. Clinicians distinguish among subtypes of insomnia based on how long the symptoms of insomnia have been present and the pattern of sleep disturbances. Although the time course and factors associated with the transition from short-term to chronic insomnia are not precisely defined, the International Classification of Sleep Disorders ${ }^{9}$ defines short-term insomnia as having a duration of less than three months. Others have used various definitions of the time at which insomnia can be considered chronic, ${ }^{2}$ but in general short-term insomnia is defined as insomnia lasting 1 to 3 months. If symptoms persist after this length of time the insomnia is considered chronic. ${ }^{9}$ Some persons with insomnia complain primarily of difficulties in getting to sleep but report that they sleep well after long sleep latencies. Other persons have difficulties in maintaining sleep and may awaken after sleeping several hours and then have difficulty returning to sleep. Although it is common to awaken during the night, most people have the ability to return to sleep once aroused. Difficulty in returning to sleep after a nocturnal awakening has been referred to as middle-of-the-night (MOTN) insomnia or as difficulty in returning to sleep insomnia. The causes of insomnia are diverse and may be associated with a biological predisposition to hyperarousal as well as situational stressors and medical disorders. ${ }^{10}$ Further, the patterns of insomnia problems may vary over time, so that it may be important to be aware that patients' primary complaints may vary over time. ${ }^{11}$

Spielman's model of the development and continuation of insomnia distinguishes among factors that predispose to, initiate or maintain sleep difficulties. ${ }^{12}$ Persons with poor sleep habits or a tendency to worry, for example, may be at greater risk for developing insomnia, but insomnia itself may occur only after experiencing an acute stressor such as job loss or change in personal relationships. This short-term insomnia occurring in response to a stressor may resolve spontaneously without treatment, especially if the stressor resolves.

If other factors arise, however, the insomnia may become chronic. Patients may become preoccupied with their sleep, for example, and worry excessively about whether they will be able to sleep and if not how the lack of sleep might affect their functioning. Excessive worry may lead to the adoption of maladaptive behaviors such as sleeping late to make up for lack of sleep, daytime napping, and going to bed early without being able to go to sleep. All of these behaviors may result in even poorer sleep, greater worry about sleep, and the continuation of a vicious cycle of sustained poor sleep.

Factors that may lead to the perpetuation of insomnia include the adoption of maladaptive strategies to cope with sleep loss (such as spending excessive time in bed) or beginning to worry excessively about whether one will be able to sleep. Worry can then lead to still greater arousal at the very time when good sleep might be facilitated by relaxation. A vicious circle of worry and excessive time in bed may lead to even poorer sleep quality and the initial insomnia may become chronic.

MOTN insomnia is not precisely defined, but can generally be considered as persistent awakening after having fallen asleep with difficulty in returning to sleep. A variety of factors may contribute to MOTN insomnia. Environmental disturbances, a restless or noisy bed partner, and need to use the toilet all may cause someone to awaken after having fallen asleep. Since the awakening may occur after several hours of sleep, sleep drive may be at a low level, making the return to sleep more difficult. Emotional reactions to having awakened, including upset at the cause of the awakening, can also contribute. Faulty sleep hygiene, such as leaving the television or radio on while falling asleep is still another potential contributor to MOTN insomnia. A careful sleep history is important in understanding patients' complaints of both short-term and MOTN insomnia.

\section{Intervening in the insomnia process}

If one accepts the behavioral model of the factors related to the development of insomnia and its transition from to chronic or insomnia proposed by Spielman and colleagues, ${ }^{12}$ a question logically arises about the optimal means of treating short-term insomnia. Possible strategies might range from watchful waiting, as it is likely that a substantial number of persons with short-term insomnia may have complaints that improve with time. An alternative might be to intervene aggressively in those with short-term insomnia in order to prevent the development of chronic insomnia. If clinicians were able to determine when and how to intervene in the transition from acute to chronic insomnia, a substantial number of cases of chronic insomnia might be prevented from developing. Unfortunately, little information has accrued on the best ways to identify at-risk individuals and intervene in the process. One study suggested that it may be possible to identify vulnerable individuals with a 
questionnaire that assesses the effect of stress on their sleep. ${ }^{13}$ This form of secondary prevention might establish the utility of heightened surveillance of persons at risk for developing chronic insomnia and justify more aggressive interventions in primary care.

\section{Treatment options for short-term insomnia}

Treatment options for short-term insomnia include both behavioral and pharmacologic interventions. Cognitive behavioral treatment of insomnia has demonstrated efficacy, ${ }^{14-16}$ but its use is limited by the availability of qualified practitioners. Many clinicians have few options for employing behavioral interventions for insomnia and may have difficulty identifying qualified persons to whom interested patients can be referred. Limited reimbursements for behavioral interventions for sleep disorders may also limit the willingness of practitioners to provide these services. ${ }^{17}$ Although behavioral treatments thus are effective for insomnia, the limited number of qualified practitioners limits the availability of this treatment in many situations. For many clinicians, the most readily available treatment for short-term insomnia may be pharmacotherapy.

One of the most common interventions for short-term insomnia is thus pharmacotherapy with a hypnotic agent. Currently available therapies include benzodiazepines, nonbenzodiazepine GABAergic medications, the melatonin receptor agonist ramelteon, and sedating antidepressants. ${ }^{18}$ Many patients report use of over-the-counter medications most of which contain antihistamines but evidence suggests that while the sedating effect of antihistamines may be helpful for a few days it becomes indistinguishable after several days. ${ }^{19}$ Probably the most commonly used sedating antidepressant is trazodone, but evidence for its efficacy is limited and it may have significant adverse effects. ${ }^{20}$

Benzodiazepines are often used to treat short-term insomnia but clinicians may be concerned about the possible development of tolerance in some patients. Little specific information is available to guide the choice of one agent over another in short-term insomnia, although clinician preferences and concerns about the development of dependence may be important factors in medication selection. Clinicians are increasingly likely to prescribe one of the nonbenzodiazepine GABAergic medications such as zolpidem, eszopiclone, or zaleplon in preference to benzodiazepines. Although effective and safe pharmacologic treatments are available for insomnia, the prescribing information for most medications such as zolpidem include the recommendation that it be taken only when the user will be able to devote 8 hours or more to sleep. This recommendation arises from the potential residual daytime sedating effects of medications for insomnia. In addition, most current pharmacotherapeutic agents do not have demonstrated efficacy in promoting sleep maintenance, although formulations of zolpidem and eszopiclone may be useful in promoting sleep throughout the night. ${ }^{21}$

As noted, MOTN insomnia arises in individuals who may have little or no difficulty in initiating sleep but who awaken after several hours and then have difficulty returning to sleep. As noted above, a significant number of persons may complain of nocturnal awakenings followed by protracted wakefulness. A challenge in treating this prevalent form of insomnia arises from the length of action of medications typically used for insomnia, including benzodiazepines such as temazepam or nonbenzodiazepine GABAergic agents such as zolpidem. These medications have half-lives that range from 1.4 to 4.5 hours for zolpidem to more than 20 hours for several of the benzodiazepines. Even those medications with short half lives may have significant residual daytime sedating effects when used for MOTN insomnia.

Sleep medications may have significant residual daytime effects that affect cognitive skills such as reaction time, motor coordination and memory. ${ }^{22}$ These skills may be particularly relevant to driving an automobile, a skill that may often be used by insomnia patients in the morning after having taken a medication. Several studies have shown that medications such as zolpidem may have significant negative impacts on real-world driving ability after having been taken at night. ${ }^{23,24}$ It is thus unsurprising that several studies have used driving as a criterion for evaluating the morning after effects of insomnia medication use.

In one study, both the long half-life drug flurazepam and the shorter half life drug temazepam had significant effects on driving 12 hours after they had been taken. Betts and Birtle ${ }^{25}$ evaluated the effect of single doses of either flurazepam $15 \mathrm{mg}$ or temazepam $20 \mathrm{mg}$ administered in counter-balanced order on driving performance on a closed course. The driving performance of 12 participants, all of whom were women, was evaluated in the morning between 9.00 and 11.00 AM, 12 hours after they had taken the drug. Participants in each condition displayed substantially more driving errors in driving over a course with barriers that required weaving or the equivalent of changing lanes. These results thus show that residual daytime effects on driving 
performance may be present even with the relatively shorter half-life medication temazepam.

Some evidence has accumulated for similar residual effects of the non-benzodiazepine drugs. In a controlled environment, Partinen et $\mathrm{al}^{26}$ gave zolpidem $10 \mathrm{mg}$, temazepam $20 \mathrm{mg}$, or placebo to women with insomnia at 2.00 AM and studied their performance in a driving simulator at 7.30 AM, 5.5 hours after taking the medication. A battery of computer-administered neuropsychological measures was completed in the morning and polysomnographies (PSGs) were done each night. Participants were 23 women with primary insomnia as defined as having a) complaint of difficulty in initiating or maintaining sleep, or of nonrestorative sleep, for 1 month; b) the sleep disturbance or related daytime fatigue caused clinically significant impairment, and c) the sleep disturbance did not occur in the context of other disorders. All were between 35 and 60 years of age (mean age 49.5 years), had driver's licenses for at least 5 years, and reported driving at least $5000 \mathrm{~km}$ each year.

The primary endpoint for the study was the mean time to a collision in the driving simulator, a variable that reflected sustained attention and reaction time in the driving simulator. No group differences were found across conditions (placebo, zolpidem, temazepam) in mean time to a collision. In secondary endpoints, neither reaction time nor memory score showed significant group differences, however, a significant effect was found for zolpidem in a measure of how well the person was able to maintain lane control over $100 \mathrm{~km}$ of simulated driving. Although these group results suggest little effect of the drugs on driving and memory performances, the authors of this study note that they observed substantial interindividual differences in performance that were possibly clinically significant. Several patients had simulator accidents after taking temazepam and zolpidem. The total number of accidents in the driving simulator, for example, was 6 for participants in the drug conditions but only 1 in the baseline and placebo conditions. Several participants thus showed much worse performance in the simulator after drug administration, suggesting that some individuals make be substantially impaired even 5.5 hours after ingestion of either temazepam or zolpidem. Clinicians should therefore be alert to the possibility that some individuals may be highly susceptible to residual effects of sleep medications and exercise appropriate caution in prescribing them for patients who may have to engage in complex mental activities the morning after taking them.

Another study ${ }^{27}$ compared the residual daytime sedating effects of either zaleplon $10 \mathrm{mg}$ or zolpidem $10 \mathrm{mg}$ after MOTN awakening. Thirty-seven adults with insomnia (mean age 44 years) received either a medication or placebo four hours after bedtime, and residual sedation was evaluated by sleep latency tests at hourly intervals after they awakened 4 hours later and for up to 7 hours after taking the medication. Residual effects of the medications were assessed through repeated sleep latency tests as well with self-reports of concentration ability and the Digit Symbol Substitution Test (DSST). The DSST is a paper and pencil test that requires individuals to rapidly substitute numbers in a series of boxes according to a key while their performance is timed. It is often considered a measure of psychomotor speed and attention that is sensitive to the effects of a sedating medication. Persons receiving the $10 \mathrm{mg}$ dose of zolpidem showed significant effects on the sleep latency test, self-report of concentration, and DSST compared to zaleplon $10 \mathrm{mg}$ and placebo for up to 7 hours after having received the drug. These results thus suggest that a standard dose of zolpidem $(10 \mathrm{mg})$ may be associated with significant cognitive effects as long as seven hours after taking it.

Currently-available treatments for insomnia thus may pose significant risks for individuals' alertness, psychomotor speed, and driving performance the morning after they are taken. These effects may be present in susceptible individuals for as long as 7 hours after taking a drug such as zolpidem, and it is possible that residual effects may be present for even longer periods if longer half life medications are used. It is thus clear that although currently available medications are clearly efficacious in promoting sleep onset in individuals with insomnia and may be useful in improving sleep maintenance, they may have lingering effects the morning after they are taken that have important implications for patients' function and safety. The need for new agents that improve sleep while having little next-morning cognitive impairment are clearly needed. ${ }^{21}$

\section{Treatment options for MOTN insomnia}

Treatment options for MOTN insomnia include both behavioral and pharmacologic interventions. Hoch et al for example, showed that sleep restriction could improve sleep continuity in older persons, decreasing MOTN awakenings. Other studies have been consistent in suggesting that the behavioral technique of sleep restriction can improve sleep continuity. In this strategy, the patient is asked to keep a sleep diary for several weeks in order to determine the amount of time spent in bed and the amount of time actually spent in sleep. The patient is then asked to spend slightly less time in bed than he or she is usually asleep. This treatment strategy 
has been shown to decrease MOTN awakenings in studies of chronic insomnia ${ }^{14}$ and might be expected to be effective in other contexts as well. As noted above, however, the limited availability of qualified persons to deliver behavioral sleep medicine services is an important drawback so that pharmacotherapy will continue to be an important option for MOTN insomnia as well.

Given the problems with residual daytime sedation and the possible effects of the time required for oral doses of sleep medications to take effect, the usefulness of several novel formulations of sleep medications have been investigated. ${ }^{28}$ Given the possibility that sublingual administration might be associated with more rapid onset of action of zolpidem, several studies have evaluated this route of administration for this medication.

Staner et $\mathrm{al}^{29}$ investigated the utility of a sublingual formulation of zolpidem using it to assist in sleep initiation after participants had napped during the day. In this model of insomnia, having participants nap in the afternoon before later trying to reinitiate sleep served to reduce sleep drive so that the they would have difficulty in falling asleep. In a group of 21 healthy volunteers with a mean age of 26.7 years, 2 doses of sublingual zolpidem ( 5 and $10 \mathrm{mg}$ ) were compared to a standard oral formulation of zolpidem $(10 \mathrm{mg})$. Study results showed that the sublingual $10 \mathrm{mg}$ dose produced shorter latencies to persistent sleep compared to the standard oral dose (12.8 vs 18.4 minutes) with similar effects on sleep onset latency and latency to stage one sleep. No significant group differences were observed for sleep maintenance variables or on subjective measures of sleep quality and all side effects were moderate or mild and resolved. These authors conclude that a sublingual formulation of zolpidem may be clinically useful given the reduction in time to sleep onset observed in this study.

Roth and colleagues ${ }^{30}$ evaluated the pharmacokinetic and pharmacodynamic characteristics of low-dose sublingual zolpidem. In this study, 24 participants (mean age 34.7 years, 13 men and 11 women) who were healthy and without sleep complaints completed a double-blind, placebo-controlled crossover trial of the daytime sedating effects of 3 doses of sublingual zolpidem $(1.0,1.75$, and $3.5 \mathrm{mg})$. The purpose of this study was thus to evaluate the sedating effects of several doses of zolpidem during the day, provide data on the time course of objective and subjective effects of the medication, and allow an investigation of the pharmacokinetic characteristics of these doses and their relation of the effects of the medication to its blood levels. Daytime sedation was measured objectively by the Digit Symbol Substitution Test (DSST) and subjectively via self-report ratings of sedation on a visual analog scale (VAS). Memory for words and reaction time were also measured to provide additional objective evaluations of the cognitive effects of the medication. Blood samples for pharmacokinetic evaluations were collected prior to the initial dose and for periods up to 12 hours after each dose.

Results of this study showed that both the $1.75 \mathrm{mg}$ and $3.5 \mathrm{mg}$ dose, but not the $1.0 \mathrm{mg}$, of sublingual zolpidem showed significant sedating effects on the DSST at 20 minutes after drug administration. The effect of the $3.5 \mathrm{mg}$ dose was substantially greater than that of the $1.75 \mathrm{mg}$ dose on this measure. Significant effects of the medication compared to placebo lasted up to 90 minutes, although participants' DSST scores did not return to levels essentially identical to those in the placebo group until 180 minutes after drug administration. Subjective reports of sedation paralleled results of objective testing, although participants' reports of sedation did not return to baseline levels until 300 minutes after drug administration. The $3.5 \mathrm{mg}$ dose showed maximum effects on reaction time at 20 minutes, while the $1.75 \mathrm{mg}$ and $1.0 \mathrm{mg}$ doses showed maximum change at 1 hour. For the two lower doses, the observed change in reaction time was not statistically significantly different from placebo. On the word recall measure, the time of maximal change from placebo was 20 minutes for the $3.5 \mathrm{mg}$ dose but 1 hour for the 1.75 and $1.0 \mathrm{mg}$ doses. Here again, the changes from baseline for the lower two doses were not significantly different from placebo levels. Pharmacokinetic analyses showed that for these participants the maximal drug concentration and areas under the curve were proportional to the dose. The half-life of zolpidem was slightly longer than 2.3 to 2.5 hours, with a time to maximal concentration of 36 to 38 minutes.

A pharmacokinetic study with elderly persons was presented by Krystal et al (reported in Lankford ${ }^{31}$ ). In that study, the pharmacokinetics of sublingual zolpidem were studied in healthy elderly volunteers. In the elderly, at the $3.5 \mathrm{mg}$ dose both the maximum concentration and the area under the curve were elevated relative to younger adults. The $1.75 \mathrm{mg}$ dose in the elderly resulted in maximum concentrations and areas under the curve that were somewhat lower than those found in younger adults. The elimination half-life, however, was similar for older as well as younger persons. Lankford suggests that this finding supports the recommendation that the lower dose of sublingual zolpidem may be most appropriate as a starting point for older persons. $^{31}$

In another study, Roth and colleagues ${ }^{32}$ report a randomized, double-blind, placebo-controlled, three-way 
crossover trial of sublingual zolpidem tartrate in 82 adults (mean age $=45.9$ years; 24 men and 58 women) with a DSM-IV diagnosis of primary insomnia and a history of MOTN insomnia with an average of 2.2 awakenings per night. Individuals were eligible to participate if, in a preliminary evaluation, they showed PSG evidence of prolonged time in returning to sleep after a scheduled MOTN awakening. Individuals participated in three treatment episodes consisting of two consecutive nights of dosing with placebo, sublingual zolpidem $1.75 \mathrm{mg}$, or sublingual zolpidem $3.5 \mathrm{mg}$. Dose of placebo or medication was administered after awakening the participants four hours after initial lights out (without regard to the participant's sleep stage at time of awakening). Treatment episodes were separated by a 5- to 12-day washout period and delivered in a randomization sequence in which all participants received each treatment condition. The primary efficacy variable was the participant's average latency to persistent sleep after the MOTN awakening.

Study results showed significant effects of both doses of sublingual zolpidem on key sleep parameters. Both doses of zolpidem were associated with a significant decrease in PSG-measured latency to persistent sleep (from a mean of 28.1 minutes for placebo to 16.9 and 9.7 minutes for the 1.75 $\mathrm{mg}$ and $3.5 \mathrm{mg}$ doses of zolpidem, respectively) as well as in patient-reported sleep onset latency. Both doses of zolpidem were associated with significant increases in total sleep time both as measured by PSG and patient report. The $3.5 \mathrm{mg}$ dose of zolpidem was associated with significantly better ratings of sleep quality in comparison to placebo, as well as in ratings of level of refreshed sleep and ability to function the next day.

This study included evaluation of the objective and subjective effects of zolpidem treatment on morning functioning. Participants completed the DSST and a visual analog scale (VAS) asking for self-report of level of sedation on each morning of the treatment periods. Results of this evaluation showed that neither DSST performance nor the VAS ratings differed significantly across conditions five hours after administration of zolpidem or placebo. It may be noted, however, that subjects reported some subjective sedation up to 5 hours after medication administration. Differences between groups at 5 hours, however, were not significantly different. It is thus not clear precisely how long patients must sleep to avoid any subjective morning sedation. No serious side effects were noted in this study, although 14 of the 82 participants reported at least one adverse event during the study. All adverse events were mild and of short duration, allowing the authors to suggest that sublingual zolpidem may be a safe and effective treatment for MOTN insomnia.

\section{Conclusion}

Studies of sublingual zolpidem thus show that this form of the drug is likely to be effective in short-term insomnia. This dosing form's rapid onset of action may confer benefits for patients taking the medication at typical bedtimes, as shown in the study by Staner et al. ${ }^{29}$ Standard doses may be associated with clinically significant residual daytime sedation so that standard doses may not be safely used in patients with MOTN insomnia. At lower doses, however, the residual daytime effects of zolpidem may be reduced and it may thus be useful in MOTN insomnia. It should be noted, however, that at least one study suggested that the subjective sedating effects of zolpidem persisted as long as five hours after dosing, a factor that may be important depending on time of administration and time of arising. Still, although standard 5 and $10 \mathrm{mg}$ doses may be associated with morning sedation if taken after midnight, ${ }^{27}$ lower doses of sublingual zolpidem may be less likely to cause morning sedation and may thus be useful for treating short-term insomnia characterized by difficulty in initiating sleep and in MOTN insomnia.

\section{Disclosure}

The author reports no conflicts of interest.

\section{References}

1. Roth T. Insomnia: definition, prevalence, etiology, and consequences. J Clin Sleep Med. 2007;3:S7-S10.

2. NIH Statement. National institutes of health state of the science conference statement. Sleep. 2005;28:1049-1057.

3. National Sleep Foundation. 2009 Sleep in America Poll. Washington, DC: National Sleep Foundation; 2009.

4. Ozminkowski RJ, Wang S, Walsh JK. The direct and indirect costs of untreated insomnia in adults in the United States. Sleep. 2007;30: 263-273.

5. Ustinov Y, Lichstein KL, Wal GS, Taylor DJ, Riedel BW, Bush AJ. Association between report of insomnia and daytime functioning. Sleep Med. 2010;11:65-68.

6. Pigeon WR, Hegel M, Unutzer J, et al. Is insomnia a perpetuating factor for late-life depression in the IMPACT cohort? Sleep. 2008;31: 481-488.

7. Perlis ML, Smith LJ, Lyness JM, et al. Insomnia as a risk factor for onset of depression in the elderly. Behav Sleep Med. 2006;4:104-113.

8. Dew MA, Hoch CC, Buysse DJ, et al. Healthy older adults' sleep predicts all-cause mortality at 4 to 19 years of follow-up. Psychosom Med. 2003;65:63-73.

9. American Academy of Sleep Medicine. International Classification of Sleep Disorders, 2nd ed. Westchester, IL: American Academy of Sleep Medicine; 2005.

10. Sateia MJ, Pigeon WR. Identification and management of insomnia. Med Clin North Am. 2004;88:567-596, vii.

11. Hohagen F, Kappler C, Schramm E, et al. Prevalence of insomnia in elderly general practice attenders and the current treatment modalities. Acta Psychiatr Scand. 1994;90:102-108. 
12. Spielman AJ, Caruso LS, Glovinsky PB. A behavioral perspective on insomnia treatment. Psychiatr Clin North Am. 1987;10:541-553.

13. Drake C, Richardson G, Roehrs T, Scofield H, Roth T. Vulnerability to stress-related sleep disturbance and hyperarousal. Sleep. 2004;27: 285-291.

14. Morin CM, Vallieres A, Guay B, et al. Cognitive behavioral therapy, singly and combined with medication, for persistent insomnia: a randomized controlled trial. JAMA. 2009;301:2005-2015.

15. Jacobs GD, Pace-Schott EF, Stickgold R, Otto MW. Cognitive behavior therapy and pharmacotherapy for insomnia: a randomized controlled trial and direct comparison. Arch Intern Med. 2004;164:1888-1896.

16. Sivertsen B, Omvik S, Pallesen S, et al. Cognitive behavioral therapy vs zopiclone for treatment of chronic primary insomnia in older adults: a randomized controlled trial. JAMA. 2006;295:2851-2858.

17. Perlis ML, Smith MT. How can we make CBT-I and other BSM services widely available? J Clin Sleep Med. 2008;4:11-13.

18. Richardson GS. Future directions in the pharmacologic treatment of insomnia. MedscapeCME. Neurology Neurosurgery. 2009; http://cme. medscape.com/viewarticle/710187.

19. Richardson GS, Roehrs TA, Rosenthal L, Koshorek G, Roth T. Tolerance to daytime sedative effects of $\mathrm{H} 1$ antihistamines. $J$ Clin Psychopharmacol. 2002;22:511-515.

20. Mendelson WB. A review of the evidence for the efficacy and safety of trazodone in insomnia. J Clin Psychiatry. 2005;66:469-476.

21. Rosenberg RP. Sleep maintenance insomnia: strengths and weaknesses of current pharmacologic therapies. Ann Clin Psychiatry. 2006;18: $49-56$.

22. Alford C, Verster J. NICE review: not nice for patients! $J$ Psychopharmacol. 2005;19:129-132.

23. Vermeeren A, Danjou PE, O'Hanlon JF. Residual effects of evening and middle-of-the-night administration of zaleplon 10 and $20 \mathrm{mg}$ on memory and actual driving performance. Hum Psychopharmacol. 1998;13:S98-S107.
24. Verster JCP, Volkerts ERP, Schreuder AHCM, et al. Residual effects of middle-of-the-night administration of zaleplon and zolpidem on driving ability, memory functions, and psychomotor performance. J Clin Psychopharmacol. 2002;22:576-583.

25. Betts TA, Birtle J. Effect of two hypnotic drugs on actual driving performance next morning. BMJ. 1982;285:852.

26. Partinen M, Hirvonen K, Hublin C, Halavaara M, Hiltunen H. Effects of after-midnight intake of zolpidem and temazepam on driving ability in women with non-organic insomnia. Sleep Med. 2003;4: 553-561.

27. Zammit GK, Corser B, Doghramji K, et al. Sleep and residual sedation after administration of zaleplon, zolpidem, and placebo during experimental middle-of-the-night awakening. J Clin Sleep Med. 2006;2:417-423.

28. Owen RT. Novel zolpidem formulations. Drugs Today (Barc). 2009;45:395-400.

29. Staner L, Eriksson M, Cornette F, et al. Sublingual zolpidem is more effective than oral zolpidem in initiating early onset of sleep in the post-nap model of transient insomnia: a polysomnographic study. Sleep Med. 2009;10:616-620.

30. Roth T, Mayleben D, Corser BC, Singh NN. Daytime pharmacodynamic and pharmacokinetic evaluation of low-dose sublingual transmucosal zolpidem hemitartrate. Hum Psychopharmacol. 2008;23:13-20.

31. Lankford A. Sublingual zolpidem tartrate lozenge for the treatment of insomnia. Expert Rev Clin Pharmacol. 2009;2:333-337.

32. Roth T, Hull SG, Lankford DA, Rosenberg R, Scharf MB. Low-dose sublingual zolpidem tartrate is associated with dose-related improvement in sleep onset and duration in insomnia characterized by middle-of-the-night (MOTN) awakenings. Sleep. 2008;31: $1277-1284$.
Nature and Science of Sleep

\section{Publish your work in this journal}

Nature and Science of Sleep is an international, peer-reviewed, open access journal covering all aspects of sleep science and sleep medicine, including the neurophysiology and functions of sleep, the genetics of sleep, sleep and society, biological rhythms, dreaming, sleep disorders and therapy, and strategies to optimize healthy sleep. The journal welcomes

\section{Dovepress}

original research, clinical \& epidemiological studies, reviews \& evaluations, case reports and extended reports. The manuscript management system is completely online and includes a very quick and fair peerreview system, which is all easy to use. Visit http://www.dovepress.com/ testimonials.php to read real quotes from published authors. 